1-day interval. ICC models were used to determine the test-retest reliability. Berg Balace Scale (BBS) was used as the reference standard to establish concurrent validity.

Results: All tests showed good to excellent reliability (ICC >0.94) except SLST which showed moderate to good reliability $(I C C=0.74)$. Three of the tests (SLST, 10 MWT, 5x SST) found to have good to excellent validity $(r>0.75)$ and three $(2$ MWT, TUG, FRT) found to have moderate to good reliability $(r>0.69)$. Results are presented in details at Table 1 and Table 2.

Conclusions: TUG, 10MWT, SLST, FRT, 5x SST and 2 MWT are reliable and valid outcome measures, and could be used to assess balance and fall risk in patients with TKA. MDC scores presented in this study can be used to evaluate change in performance over time or effectiveness of interventions. 2 MWT and $5 x$ SST were determined as the most reliable and valid methods among the investigated tests.

Disclosure of Interest: None declared

DOI: 10.1136/annrheumdis-2017-eular.5918

\section{FRI0737-HPR THE SOURCES OF PAIN IN ABDOMEN IN PATIENTS WITH RHEUMATOID ARTHRITIS}

H. Palhuyeva ${ }^{1}$, A. Litviakov ${ }^{1}$, V. Shynkovski ${ }^{2}$, N. Podolinskay ${ }^{2} .{ }^{1}$ Internal diseases; ${ }^{2}$ Vitebsk State Order of Peoples' Friendship Medical Universityon, Vitebsk, Belarus

Background: Systemic diseases of connective tissue associated with the early development of atherosclerosis [1,2]. It is well-known that the main cause of decrease the quality of life patients with rheumatoid arthritis (RA) is a chronic pain syndrome. The cause of pain can be joint inflammatory process, systemic manifestations of RA (vasculitis, pericarditis, pleurisy, polyneuropathy), complications of drug therapy, comorbid conditions. Pain in the stomach area may be associated with NSAID-gastropathy, problems with spinal discs, and perhaps with atherosclerotic lesions of the abdominal aorta.

Objectives: To estimate atherosclerotic changes of arteries in patients with RA and determine possible sources of pain in the abdomen by ultrasound-control palpation.

Methods: We included 75 patients with RA (age 38,7 $\pm 7,4$, males 93,3\%) and 29 healthy subjects, matched for age and gender, without a history of cardiovascular diseases. An ultrasound investigation of the arterial vassal with measurement of the intima-media thickness (IMT) of carotids was performed. To determine the source of pain in the abdominal cavity ultrasound-control palpation of duodenal bulb, gallbladder, lumbar discs, and abdominal aorta was done. Severity of pain was assessed using the VAS.

Results: It has been determined, that in RA group IMT was $0.8 \mathrm{~mm}(0.7-0.9)$, compared with $0,6 \mathrm{~mm}(0,6-0,7)$ in control group. IMT positively correlate with the age, duration of disease, Ritchie index, C-reactive protein level. In $22(29,3 \%)$ patients with RA we found atherosclerotic plaques lesion in carotids, aorta, and vessels of the lower extremities. Present of atherosclerotic plaques associated with disease durations (12 years $(10-15)$ in group with plaques and 5 years $(3-8)$ in group without plaques). The presence of atherosclerotic plaques is associated with rheumatoid factor $\left(c^{2}=1,02, p>0,05\right)$, and systemic manifestations of RA $\left(c^{2}=15,89, p<0,001\right)$.

In RA group $36(48 \%)$ patients had indicated the presence of pain in the abdomen while performing ultrasound control palpation. In $15(20 \%)$ cases was detected pain during palpation of the lumbar spine, VAS 48 (36-59). In 21 (28\%) cases, patients indicated pain during palpation of the abdominal aorta, VAS 42 (31-65). In this group of patients we found signs of the atherosclerotic lesions of aorta: change in the contour of the vessel, the heterogeneous structure of the vascular wall. Atherosclerotic plaques in the abdominal aorta are founded in 21 (28\%) cases.

Conclusions: Patients with RA had an increase thickness of IMT and atherosclerotic plaques, which appear in various vascular regions. During performing ultrasound-control palpation in $36(48 \%)$ patients was detected pain, associated with vertebral changes $15(20 \%)$, and with atherosclerotic changes of abdominal aorta $-21(28 \%)$.

\section{References:}

[1] High incidence of cardiovascular events in a rheumatoid arthritis cohort not explained by traditional cardiac risk factors. I.D. del Rincon et al. Arthritis Rheum. 2001;44:2737-2745.

[2] Cardiovascular disease and risk factors in patients with rheumatoid arthritis, psoriatic arthritis, and ankylosing spondylitis. C. Han et al. J. Rheumatol. 2006;33(11):2105-2107.

Disclosure of Interest: None declared

DOI: 10.1136/annrheumdis-2017-eular.5921

\section{FRI0738-HPR QUALITY IN REHABILITATION FOR RHEUMATIC AND MUSCULOSKELETAL DISEASES (RMDS): DEVELOPMENT AND TESTING OF A RMD REHABILITATION QUALITY INDICATOR SET}

I. Johansen ${ }^{1}$, M. Klokkerud ${ }^{1}$, T. Glott ${ }^{2}$, M. Larsen ${ }^{3}$, K. Lande ${ }^{4}$, K.B. Hagen ${ }^{1}$, I. Kjeken ${ }^{1}$. ${ }^{1}$ National Advisory Unit on Rehabilitation in Rheumatology, Diakonhjemmet Hospital; ${ }^{2}$ Sunnaas Hospital; ${ }^{3}$ National Rheumatism Association, Oslo; ${ }^{4}$ Haugesund Rheumatism Hospital, Haugesund, Norway

Background: Rehabilitation in Norway is characterized by a lack of coordination across levels of care, and by large variations in content and quality. A means to monitor the quality of care is to use quality indicators (QI), which are related to structure (the settings in which care is given), process (what is actually being done) or outcome of health care.

Objectives: To develop and test a set of quality indicators for rehabilitation of people with RMDs.

Methods: The QI set was developed following the Rand/UCLA Method and pilottested for validity and feasibility in 29 specialist and primary care rehabilitation units. Pass rates were recorded in telephone interviews with managers of the rehabilitation units (structure QIs (yes/no)), or patients (process and outcome Qls (yes/no)). Time use and participants rating of face validity on a numeric rating scale $(0-10,10=$ high validity) were recorded. Separate $Q$ I pass rates and summary QI pass rates were calculated.

Results: A total of 164 patients (mean (SD) age 58.5 (16.5) years and 76\% women) participated. Mean (range) validity score for managers/patients was 8.3 (8)/7.9 (9), and mean answering time was 6.0/5.5 minutes.

A $100 \%$ summary pass rate was reached by only two of the rehabilitation units and $4 \%$ of the patients.

Conclusions: To our knowledge this is the first Ql set developed to measure QI pass rates for rehabilitation of RMDs. The results indicate that the QI set is acceptable to respondents, and it's validity and feasible format make it suitable for monitoring quality of rehabilitation. The large variety in pass rates suggests a high potential for quality improvement in rehabilitation.

Disclosure of Interest: None declared

DOI: 10.1136/annrheumdis-2017-eular.1809

\section{FRI0739-HPR A RHEUMATOLOGY PATIENTPANEL: THE PATIENT IS THE EXPERT, WE ARE THE SPECIALIST}

J.E. Voorneveld-Nieuwenhuis, M.D. Kok, N. Basoski, M. Holtrop, D. Lopes Barreto. Rheumatology, Maasstad Hospital Rotterdam, Rotterdam, Netherlands

Background: Patient participation is known in the field of rheumatology research and is becoming more customary. The patient perspective can improve the care and line specific needs of the patient to the delivered care.Recently, ourRheumatology department within the Maasstad Hospital introduced the Value Based Health Care principle in order to optimize the current healthcare system.

Abstract FRI0738-HPR - Table 1. Structure, process and outcome Quality indicators (QI) with Pass rates (PR) in \%

\begin{tabular}{|c|c|c|}
\hline STRUCTURE QI (answered by unit managers) & PR & PROCESS QI (answered by patients) \\
\hline Has a defined patient target group & 96 & \\
\hline Procedures for & & Had an initial bio-psycho-social assessment \\
\hline Developing an individual rehabilitation plan & 76 & Received a written rehabilitation plan \\
\hline Regular team meetings with patients & 72 & Participated in minimum 2 team meetings \\
\hline Meetings with possibility to participate for next of kin and external HPs* & 65 & It was possible for next of kin and external HPs to participate in the team meetings \\
\hline Individual plans for follow-up after discharge & 52 & $\begin{array}{l}\text { Received an individual plan for follow-up } \\
\text { External HPs* were involved in follow-up }\end{array}$ \\
\hline Procedures for involving patients in & & Participated in \\
\hline Setting goals & 93 & Setting goals \\
\hline Planning the intervention & 93 & Planning the intervention \\
\hline Evaluating the intervention & 89 & Planning follow-up \\
\hline Use of validated assessment instruments & 69 & OUTCOME QI (answered by patients) \\
\hline Assessment of & & Satisfied or very satisfied with rehabilitation \\
\hline Goal attainment at discharge & 41 & Reached important goals \\
\hline Goal attainment 3-6 months after discharge & 21 & \\
\hline Function at admission and discharge e & 79 & Had important improvement in physical, psychological or social function \\
\hline Function 3-6 months after discharge & 24 & \\
\hline HRQoL at admission and discharge & 29 & Had improvement in quality of life \\
\hline HRQoL 3-6 months after discharge & 17 & \\
\hline Registration of adverse events & 96 & Experienced negative events \\
\hline
\end{tabular}

${ }^{*}$ External HPs are health professionals from other health care settings, such as primary care. 
We thought to measure the value for patients we should let them participate in the project and asked them about what is of value for them and what are there goals. A good reason to start with a patient panel.

Objectives: For this reason we aimed to create a patient panel of rheumatology patients that are well informed and prepared to actively cooperate and participate in research, and co-design novel healthcare strategies.

Methods: Staff members (e.g. doctors and nurses) were asked to nominate patients that are expected to actively participate in the panel. Nominated patients were verbally approached and asked to participate. After mutual agreement a contract was signed were patients discretion was warranted, the capacity to handle confidential information was assessed and equality between members of the panel and staff was ensured. Enlargement of the panel is primarily staff-driven, but panel members are also invited to actively recruit other rheumatology patients. Results: The initial enrolment period lasted for six months. Thereafter, meetings were organized were discussion were held on various themes such as goals and value for rheumatology patients. Additionally, a focus group of rheumatoid arthritis (RA) patients was assembled to evaluate a PROMs measurement tool. To date, a number of four panel discussion have been held. Currently, our patient panel consists of 54 patients with all kinds of rheumatic diseases. Their demographic and clinical data are presented in Table 1

Table 1

\begin{tabular}{lc}
\hline & Value \\
\hline Patients (n) & 54 \\
Gender (\%male) & 17 \\
Age (yrs) & $51(20-77)$ \\
Disease duration (yrs) & $9(0-30)$ \\
Diagnosis (\%) & \\
- Rheumatoid Artrithis & 54 \\
- Arthrosis & 15 \\
- Fibromyalgia & 10 \\
- SpA (including APs and SA) & 12 \\
- Other & 9 \\
\hline
\end{tabular}

Our panel has been asked to participate in other (hospital-wide) programmes including the development and evaluation of a patient portal. We organize about three meetings for the whole panel every year and arrange focus group meetings to discuss specific subjects.

Conclusions: Panel members are very open and enthusiastic. Some quotes: "I'm happy to do something in return for the good care I receive.", and "I want to promote participation in scientific research". Deployment of patient participation for co-creating innovations alongside research is an asset these days to connect changes to patients perception.

In real live involving patient as an expert is not an effortless action for both patients and the expert care takers, it leads to satisfaction and an effective treatment.

Disclosure of Interest: None declared

DOI: 10.1136/annrheumdis-2017-eular.3454

\section{FRI0740-HPR ONLINE REMOTE MONITORING OF PATIENTS WITH RHEUMATOID ARTHRITIS: FIRST RESULTS OF A PILOT STUDY}

L. Renskers $^{1}$, S.A.A. Rongen ${ }^{1,2}$, S. Teerenstra ${ }^{3}$, M.E.J.L. Hulscher ${ }^{1}$,

P.L.C.M. van Riel ${ }^{1,2}$. ${ }^{1}$ IQ Healthcare, Radboud Institute for Health Sciences,

Radboud university medical center, Nijmegen; ${ }^{2}$ Rheumatology, Bernhoven,

Uden; ${ }^{3}$ Department for Health Evidence, Radboud Institute for Health Sciences, Radboud university medical center, Nijmegen, Netherlands

Background: Since the disease activity of patients with RA tends to fluctuate between visits and disease flares are easily missed during regular visits, patients might benefit from a more closely spaced determination of disease activity, which could be realised by iMonitor $^{1}$. iMonitor is a Software Medical Device developed and funded by Pfizer. This online system allows patients to complete three kinds of patient-reported outcome measures (PROMs): the Health Assessment Questionnaire (HAQ), Rheumatoid Arthritis Impact of Disease (RAID) and Rheumatoid Arthritis Disease Activity Index-5 (RADAI-5). iMonitor might contribute to identification of patients who need additional medical attention in between visits and reduction of visits for patients with stable disease activity. Objectives: To determine the degree to which the PROM-scores in iMonitor are associated with DAS28. Moreover, PROM preferences and completion rates were studied.

Methods: Patients were recruited at Bernhoven (Uden, the Netherlands) by an announcement on the hospital website, leaflets and meetings. Instruction classes were organised in which researchers assisted patients in using iMonitor. Patients indicated which $\operatorname{PROM}(\mathrm{s})$ they want to complete in iMonitor and chose reminder email frequency (weekly, two-, four, six, or eight-weekly). Descriptive analyses were used to describe characteristics of the study population. Scatter plots with regression equations were performed with DAS28 as dependent and PROM as independent variable to determine the association between DAS28 and PROMs. Moreover, Pearson's correlations were calculated. PROM-scores within the fourteen day window before and after DAS28 assessment were included. Results: In total 33 patients with RA were included, seventeen of them were female $(52 \%)$. Mean $( \pm S D)$ age was $56 \pm 11$ years. Seventeen patients $(52 \%)$ attended the instruction classes. Majority of patients $(n=10)$ chose all three PROMs to complete, nine patients chose RAID+RADAI-5, seven chose HAQ+RAID, three chose RAID, two chose RADAI-5 and two chose HAQ+RADAI-5. From March 2016 until December 2016, 435 RAID-values, 329 RADAl-5-values and 222 HAQ-values were gathered. When taking PROM-values within the fourteen day window before and after DAS28 assessment, 159 DAS28-values could be coupled to 320 PROM-values. Regression analysis showed the following proportions of explained variance $\left(R^{2}\right)$ : 0.17 for HAQ, 0.32 for RAID and 0.29 for RADAI-5. Pearson's correlation coefficients were 0.41 for $\mathrm{HAQ}, 0.57$ for RAID and 0.54 for RADAI-5. Most chosen reminder email frequency was four weeks ( $n=21)$. Completion rates (measured until December 31,2016 ) were $65 \%$ for patients with one week PROM-frequency and for patients with two, four, six and eight week frequency completion rates were $39 \%, 24 \%, 30 \%$ and $0 \%$, respectively.

Conclusions: RAID and RADAI- 5 were moderately associated with DAS28 and showed highest proportions of explained variance. The association between HAQ and DAS28 was weaker. Patients receiving a weekly reminder email showed highest completion rates. This pilot study is a first step towards personalised healthcare and patient involvement in online remote monitoring.

\section{References:}

[1] Medical and Educational Goods and Services (MEGS): iMonitor. 2014 Available from: http://www.pfizer.co.uk/content/medical-and-educational-goodsand-services-megs-imonitor.

Disclosure of Interest: L. Renskers Grant/research support from: Pfizer, S. Rongen: None declared, S. Teerenstra: None declared, M. Hulscher: None declared, P. van Riel Grant/research support from: Pfizer DOI: 10.1136/annrheumdis-2017-eular.5801

\section{FRI0741-HPR SCREENING FOR DEPRESSION AND ANXIETY IN AN OUTPATIENT RHEUMATOLOGY CLINIC USING VALIDATED SELF-APPLIED QUESTIONNAIRES}

L.F. Perez, A. Martinez, M. Moreno, L. Silveira. Reumatologia, Instituto Nacional de Cardiologia Ignacio Chavez, Mexico City, Mexico

Background: Anxiety and depression are often present in chronic rheumatic diseases. Recognition of these psychological disorders is fundamental for proper patient management. The absence of screening leaves more than $>50 \%$ of patients with depression unidentified. Patient Help Questionnaire-9 (PHQ-9) and General Anxiety Disorder-7 (GAD-7) are two validated self-applied questionnaires that are appropriate to assess the presence of, depression and anxiety, respectively.

Objectives: 1) To assess the prevalence of depression and anxiety in a hospital based outpatient Rheumatology clinic and 2) To provide the attending physician with appropriate instruments that allow a rapid orientation on the psychological status of her/his patient.

Methods: Consecutive patients that attended our outpatient Rheumatology clinic from March to June 2016 were invited to participate in this crosssectional study. Participants filled out PHQ-9 and GAD-7 in the waiting room. The prevalence and severity of anxiety and depression were calculated for the most prevalent diagnoses.

Results: A total of 410 patients were recruited; 339 (82.8\%) were female. Overall, $191(46.6 \%)$ patients reported depressive symptoms (PHQ-9>5). Of them, 87 $(21.2 \%)$ were classified as having moderate depression or higher (PHQ-9 $>10)$. Prevalence of depression and anxiety among study participants according to each rheumatic disease is depicted in Table 1. Prevalence of moderate or severe depression was significantly different among various rheumatic diseases $(p=0.001)$. Regarding anxiety symptoms, they were reported in $168(40.7 \%) ; 67$ $(16.2 \%)$ of them had moderate or severe anxiety.

\begin{tabular}{|l|l|l|l|}
\hline Disease & N & $\begin{array}{l}\text { Moderate or severe } \\
\text { depression }\end{array}$ & $\begin{array}{l}\text { Moderate or } \\
\text { severe anxiety }\end{array}$ \\
\hline SLE & $99(24.1 \%)$ & $16(16.2 \%)$ & $12(12.1 \%)$ \\
\hline RA & $107(26.1 \%)$ & $26(24.3 \%)$ & $17(15.9 \%)$ \\
\hline FM & $87(21.3 \%)$ & $\begin{array}{l}30(34.5 \%) \\
p=0.001\end{array}$ & $\begin{array}{l}27(31 \%) \\
p=0.001\end{array}$ \\
\hline OA & $69(16.8 \%)$ & $\begin{array}{l}25(36.2 \%) \\
p=0.001\end{array}$ & $\begin{array}{l}22(31.9 \%) \\
p=0.001\end{array}$ \\
\hline SSC & $18(4.4 \%)$ & $13(26 \%)$ & $8(16 \%)$ \\
\hline $\begin{array}{l}\text { Inflammatory } \\
\text { myopathies }\end{array}$ & $14(3.4 \%)$ & $4(28.6 \%)$ & $2(14.3 \%)$ \\
\hline Osteoporosis & $49(12 \%)$ & $14(28.6 \%)$ & $9(18.4 \%)$ \\
\hline APS & $42(10.2 \%)$ & $\begin{array}{l}1(2.4 \%) \\
p=0.001\end{array}$ & $3(7.1 \%)$ \\
\hline
\end{tabular}

Table 1. Prevalence of depression and anxiety amone study participants according to each rheumatic disease. (SLE - Systemic lupus erythematosus, RA - Rheumatoid Arthritis, FM Fibromyalgia, OA - Osteoarthritis, SSC-Systemic sclerosis, APS - Antiphospholipid syndrome.

Conclusions: This cross-sectional study shows that anxiety and depression are frequent in the Rheumatology clinic. We demonstrated that the use of a self-applied screening tool can help clinicians to properly detect depression and anxiety associated with diverse rheumatic diseases. Specia attention should be paid to patients with fibromyalgia and osteoarthritis. 